This item was submitted to Loughborough's Research Repository by the author.

Items in Figshare are protected by copyright, with all rights reserved, unless otherwise indicated.

\title{
The computer-aided design and rapid prototyping fabrication of removable partial denture frameworks
}

PLEASE CITE THE PUBLISHED VERSION

PUBLISHER

Professional Engineering Publishing / @ IMechE

VERSION

VoR (Version of Record)

LICENCE

CC BY-NC-ND 4.0

\section{REPOSITORY RECORD}

Eggbeer, Dominic, Richard J. Bibb, and R.J. Williams. 2019. "The Computer-aided Design and Rapid Prototyping Fabrication of Removable Partial Denture Frameworks". figshare.

https://hdl.handle.net/2134/5708. 
This item was submitted to Loughborough's Institutional Repository (https://dspace.lboro.ac.uk/) by the author and is made available under the following Creative Commons Licence conditions.

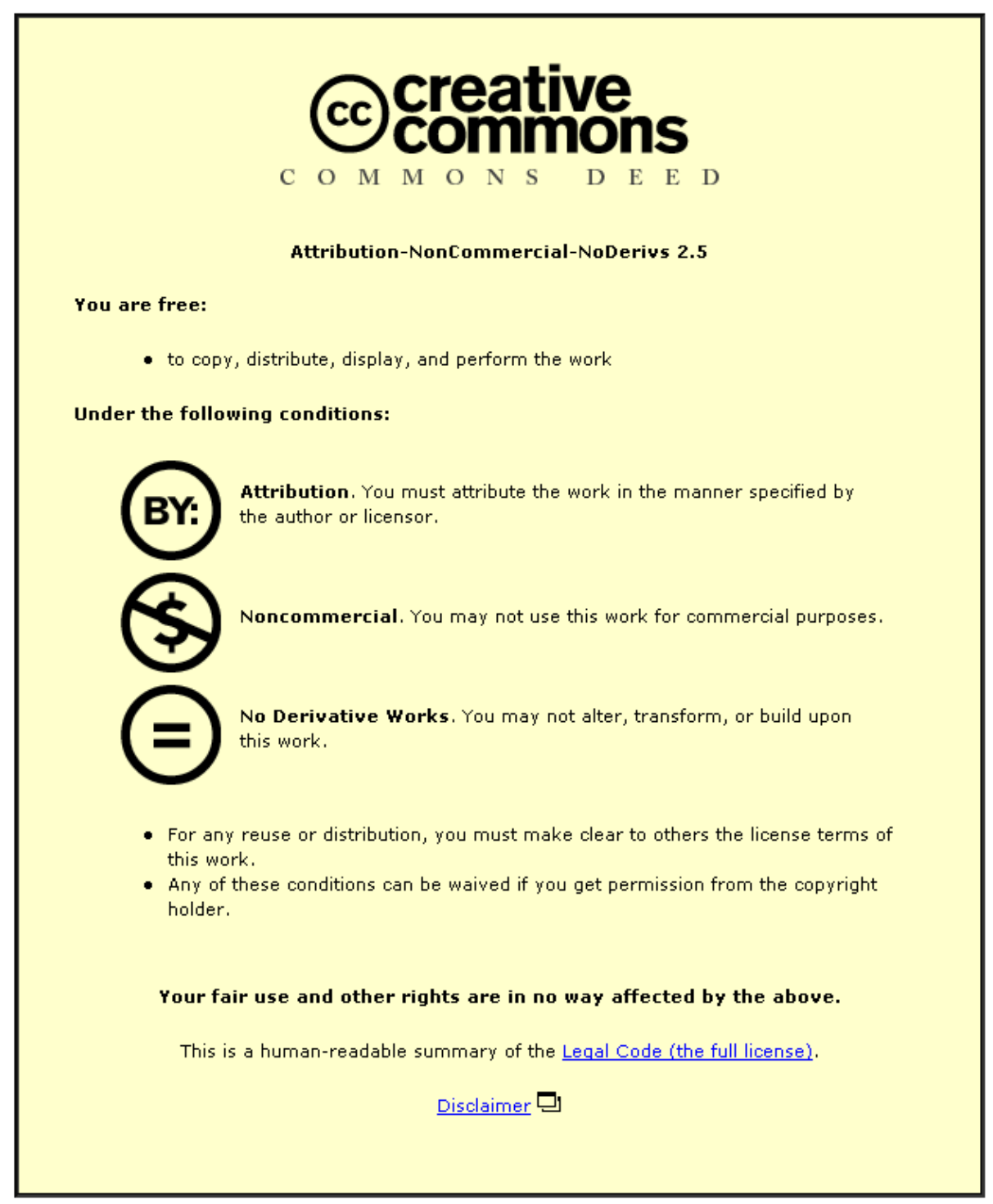

For the full text of this licence, please go to: http://creativecommons.org/licenses/by-nc-nd/2.5/ 


\title{
The computer-aided design and rapid prototyping fabrication of removable partial denture frameworks
}

\author{
D Eggbeer $^{1 *}$, R Bibb ${ }^{1}$, and R Williams ${ }^{2}$ \\ ${ }^{1}$ The National Centre for Product Design and Development Research, The University of Wales Institute, Cardiff, UK \\ ${ }^{2}$ Centre for Dental Technology, University of Wales Institute, Cardiff, UK
}

The manuscript was received on 12 August 2004 and was accepted after revision for publication on 13 January 2005.

DOI: $10.1243 / 095441105 X 9372$

\begin{abstract}
This study explores the application of computer-aided design and manufacture (CAD/ CAM) to the process of electronically surveying a scanned dental cast as a prior stage to producing a sacrificial pattern for a removable partial denture (RPD) metal alloy framework. These are designed to retain artificial replacement teeth in the oral cavity. A cast produced from an impression of a patient's mouth was digitally scanned and the data converted to a three-dimensional computer file that could be read by the computer-aided design (CAD) software. Analysis and preparation were carried out in the digital environment according to established dental principles. The CAD software was then used to design the framework and generate a standard triangulation language (STL) file in preparation for its manufacture using rapid prototyping (RP) methods. Several RP methods were subsequently used to produce sacrificial patterns, which were then cast in a chromium-cobalt alloy using conventional methods and assessed for accuracy of fit.

This work demonstrates that CAD/CAM techniques can be used for electronic dental cast analysis, preparation, and design of RPD frameworks. It also demonstrates that RP-produced patterns may be successfully cast using conventional methods and that the resulting frameworks can provide a satisfactory fit.
\end{abstract}

Keywords: computer-aided design, rapid prototyping, removable partial denture

\section{INTRODUCTION}

Computer-aided design and manufacture (CAD/ CAM) and rapid prototyping (RP) techniques have been extensively employed in the product development sector for many years and have also been extensively used in maxillofacial technology and surgery [1-3]. In addition, CAD/CAM technologies have been introduced into dentistry, particularly for the manufacture of crowns and bridges [4-6], but there has been little research into the use of such methods in the field of removable partial denture (RPD) framework fabrication. This may in part be attributed to the lack of suitable dedicated software. Recent pilot studies have showed that computeraided design (CAD) and RP methods of designing and producing a sacrificial pattern for the production

\footnotetext{
* Corresponding author: The National Centre for Product Design and Developmental Research, The University of Wales Institute, Cardiff, Western Avenue, CardiffCF5 2YB, UK. email:deggbeer-pdr@ uwic.ac.uk
}

of metal alloy components of RPD metal frameworks could have promising applications $[\mathbf{7}, \mathbf{8}]$. These studies explored the application of computer-aided technologies to the surveying of digital casts and pattern design and the subsequent production of sacrificial patterns using RP technologies.

The potential advantages offered by the introduction of advanced CAD/CAM and RP into the field of RPD framework fabrication include automatic determination of a suggested path of insertion, the almost instant elimination of unwanted undercuts (re-entry points), and the equally rapid identification of useful undercuts. At another stage, components of an RPD could be stored in a library and 'dragged and dropped' in place on a scanned and digitally surveyed cast from icons appearing on screen, allowing virtual pattern making to be carried out in a much faster time than is achieved by current techniques. The quality assurance of component design can also be built into the software. Since RP machines build the object directly, scaling factors may also be precisely imposed in order to compensate for shrinkage 
in casting. In addition to the potential time savings, the CAD/RP process also delivers inherent repeatability, which may help to eliminate operator variation and to improve quality control in the dental laboratory.

The current paper reports an investigation into the application of CAD and RP methods to achieve the stages of surveying and design using an appropriate CAD software package. It also discusses the application of RP technologies to produce sacrificial patterns for casting the definitive chromium-cobalt framework component. The advantages, limitations, and future possibilities of these techniques are concluded.

\section{MATERIALS AND METHODS}

\subsection{Three-dimensional scanning}

A three-dimensional scan of a partially dentate patient's dental cast was obtained using a structured white-light digitizer (Comet 250, Steinbichler Optotechnik GmbH, Neubeuern, Germany). This particular type of scanner is used in high-precision engineering applications and has been used in maxillofacial technology [9]. Multiple overlapping scans were used to collect point cloud data that was aligned using Polyworks software (InnovMetric Software Inc., Quebec, Canada). Spider software (Alias-Wavefront Inc., Toronto, Ontario, Canada) was used to produce a polygon-surface standard triangulation language (STL) (C. R. Manners, 1993, 'STL file format' available on request from 3D Systems Inc., Valencia, California, USA) model file that could be imported into any number of CAD software packages, including that used in this study.

\subsection{CAD of the RPD framework}

The CAD package used in this study was chosen because it is well adapted to the design of complex arbitrary shapes that are required when designing custom appliances and devices that must fit human anatomy. The software has tools analogous to those used in physical sculpting and enables a manner of working that most closely mimics that of the dental technician working in the laboratory (FreeForm ${ }^{\circledR}$, SensAble Technologies, Inc., Woburn, Massachusetts, USA). The software utilizes a haptic interface (Phantom ${ }^{\circledR}$ Desktop haptic interface; SensAble Technologies Inc.) that incorporates positioning in three-dimensional space and allows rotation and translation in all axes, transferring hand movements into the virtual environment (Fig. 1). It also allows

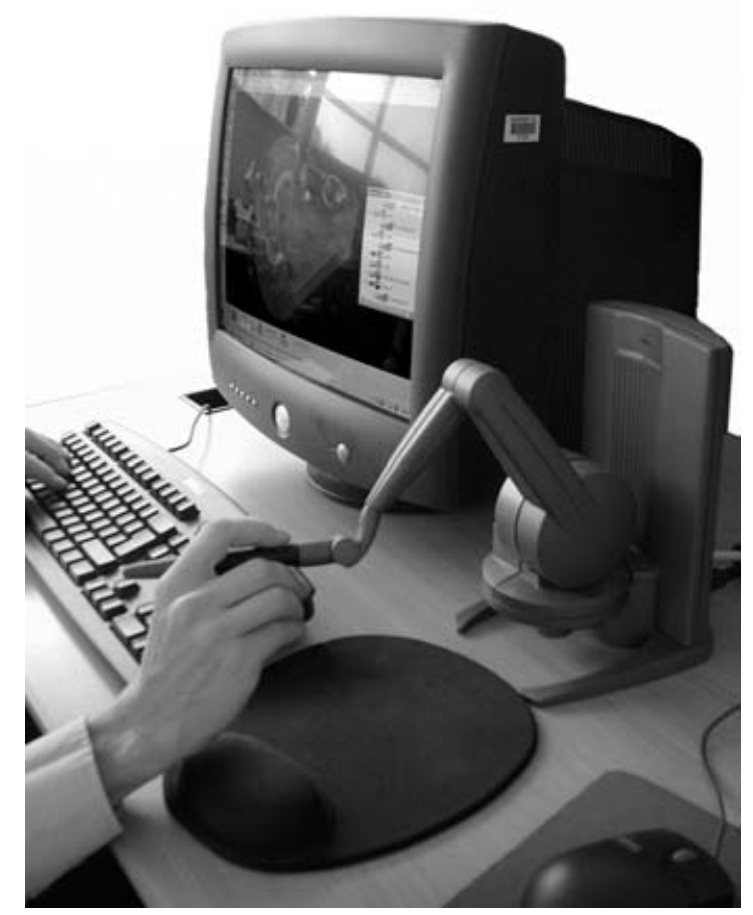

Fig. 1 The Phantom ${ }^{\circledR}$ stylus

the operator to feel the object being worked on in the software. The combination of tools and force feedback sensations mimics working on a physical object and allows shapes to be designed and modified in an arbitrary manner.

Objects being designed or worked on are referred to as virtual 'clay', which can be rotated and viewed from any angle on the screen. A 'buck' setting prevents a model from being unintentionally modified but allows 'clay' to be added or copied.

\subsection{Surveying}

Surveying is undertaken in dental technology laboratories to identify useful dental features in order for the RPD design to be retained in the oral cavity effectively. Dental surveying identifies areas of undercut present on the patient's teeth and soft tissue. The effect is similar to analysing a product design to find the split line for a two-part mould.

Like many CAD packages, the CAD software used in this study has an automatic 'parting line' (also known as a 'split line') function, which was used to delineate up and down facing surfaces, thus identifying areas of undercut in a different colour from the 'buck' model. The effect is identical with the physical technique of using dental survey lines to identify and mark the most bulbous areas of teeth with a pencil line (highlighted in Fig. 2a). The undercuts were assessed in order to establish the best path 


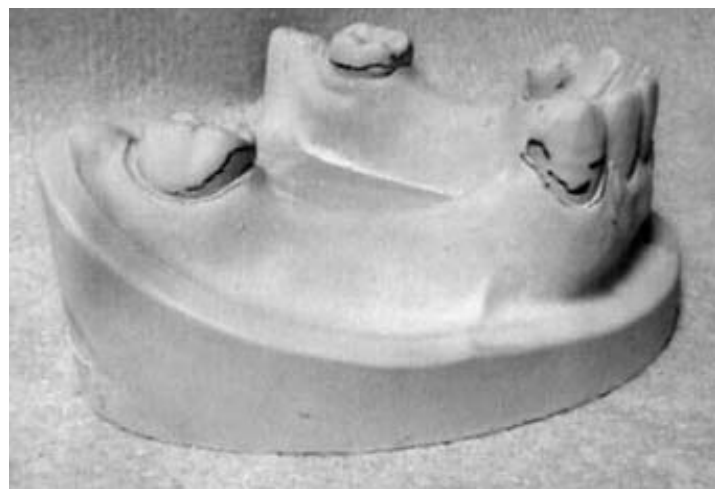

(a)

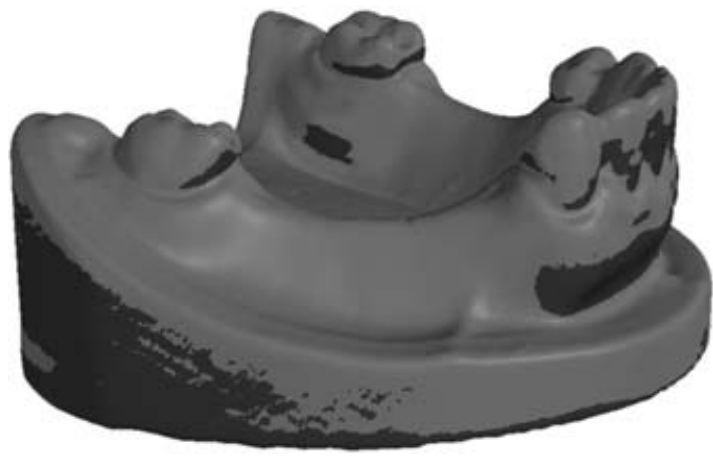

(b)

Fig. 2 (a) The physically surveyed cast. (b) The digitally surveyed 'buck' cast. Undercuts are shown as dark areas

of insertion and possible points for active clasp termination and the model rotated accordingly.

A visual comparison (Figs $2 a$ and $b$ ) was made between the physically surveyed cast and the same model cast surveyed using the software.

Once a suitable angle was chosen, the model was re-exported as an STL file.

\subsection{Removing unwanted undercuts}

When creating an RPD most undercuts are removed so that the resulting framework can be inserted and removed in a comfortable manner. The STL file of the rotated cast was imported into FreeForm ${ }^{\circledR}$, but this time using the 'extrude to plane' option. When the cast was viewed from above, this option took the maximum extents of the profile and extruded them down by a user-defined distance. This effectively removed undercuts and replaced them with vertical surfaces (Figs $3 a$ and $b$ ).

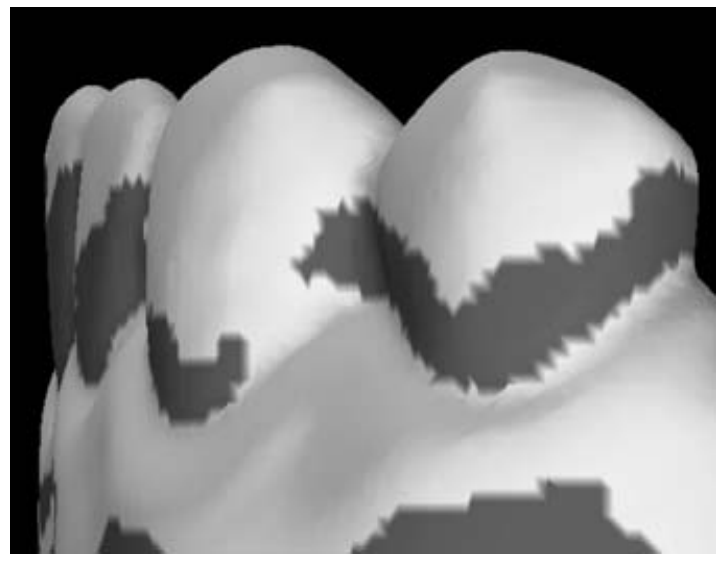

(a)

\subsection{Identifying useful undercuts}

FreeForm's 'ruler' tool was used to measure the distance between the original cast model and the version with undercuts removed. The useful undercuts were marked with a line for use in the design stages. RPDs provide firm location on the existing dentition by using flexible clasps. The clasp components of the RPD open on initial contact during insertion and removal and return to their original position within the undercut on final seating, thus providing secure retention.

\subsection{Creation of relief}

The areas without teeth require a spacer, known as relief to prevent the framework from resting on the surfaces of the soft tissues. Relief was created by selecting and copying an area from the cast with undercuts removed, and then pasting this as a new piece

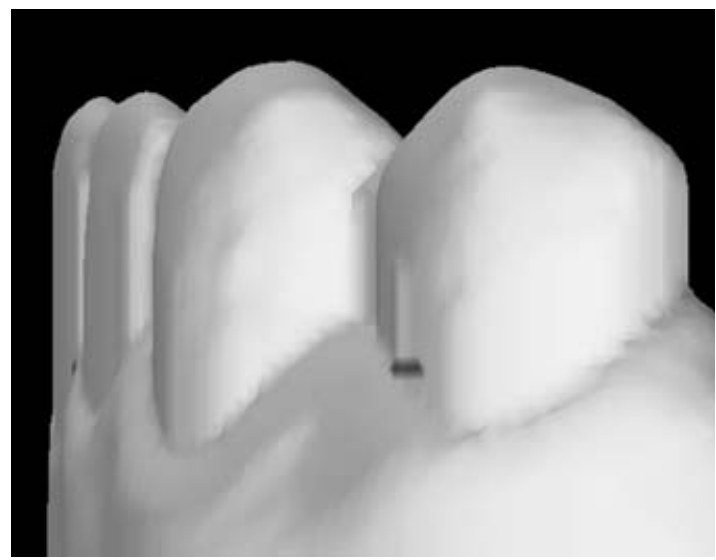

(b)

Fig. 3 (a) Undercuts are shown as dark areas. (b) Undercuts have been removed and replaced by vertical surfaces 
of clay. This was then offset to the outside by $1 \mathrm{~mm}$. The results of this process are highlighted in Fig. 4.

The entire modified model was saved as an STL file and then re-imported using the 'buck' setting to avoid unintentional modification during the next stages of RPD design.

\subsection{Framework design}

The RPD design employed in this study was based on recognized dental technology methods emphasizing simplicity, aesthetics, and patient comfort [10]. Some of the key design features outlined in the design stages are labelled in Fig. 5.

The entire framework was designed on the relieved 'buck' cast with undercuts removed, with the exception of the clasp components. The clasps use the undercuts to function and were therefore designed on the original 'buck' cast. The following techniques were used in the framework design.

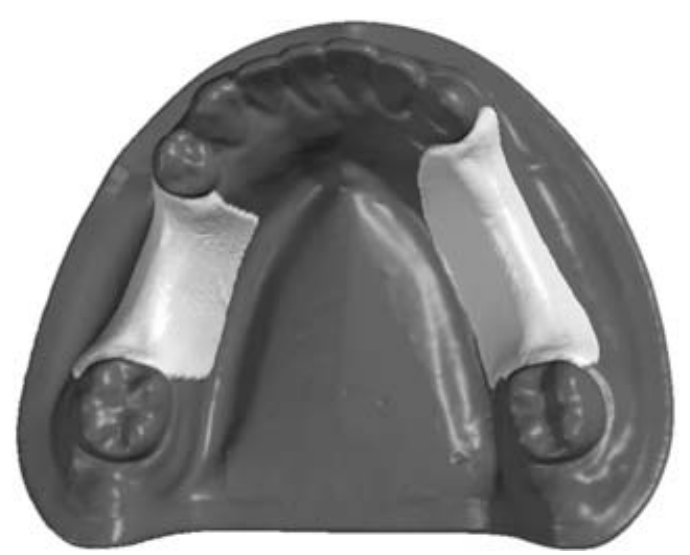

Fig. 4 Relieved edentulous areas shown in the lighter colour on the dark cast

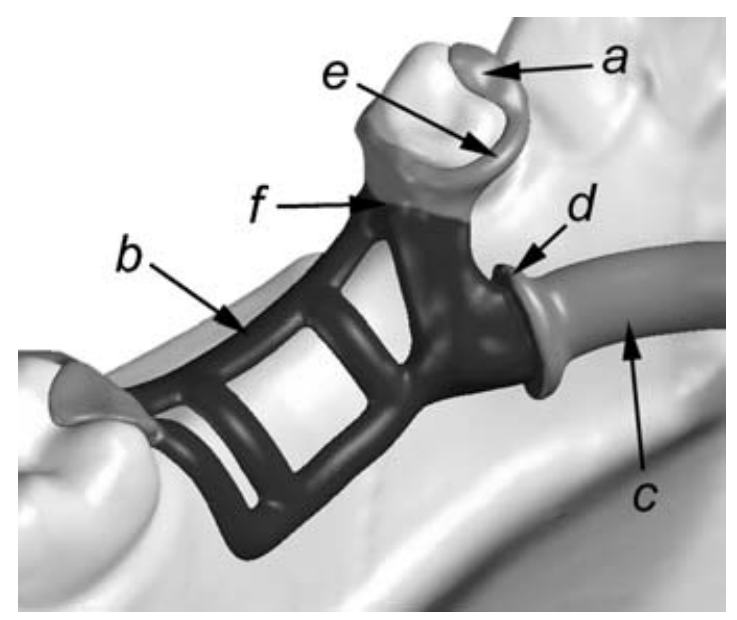

Fig. 5 Key design features: a, occlusal rest; b, polymeric retention frame; $c$, lingual bar; $d$, acrylic line; e, non-active clasp; $\mathrm{f}$, guide plate

\subsubsection{Occlusal rests ( $\mathrm{a}$ in Fig. 5)}

A combination of two-dimensional drawing and three-dimensional creation and manipulation tools was used to create pieces of clay that were copied and located where required on the teeth.

\subsubsection{Polymeric retention framework (b in Fig. 5), lingual bar (c in Fig. 5), acrylic line (d in Fig. 5), and non-active clasps (e in Fig. 5)}

The 'draw' tool was used to locate curves directly on to the cast surface. These formed the centre of the framework's profile (Fig. 6). The 'groove' tool was used to define and create the exact oval and square sectional dimensions as clay.

\subsubsection{Guide plates ( $\mathrm{f}$ in Fig. 5)}

Guide plates were created using the same method as relief creation. The 'attract' and 'smudge' tools were also used to build up plate areas and to blend them onto the framework sections.

\subsubsection{Finishing}

'Smooth', 'attract', and 'smudge' tools were used to blend the components together. The 'buck' cast was removed, acting as a Boolean cutting tool to leave just the clay framework.

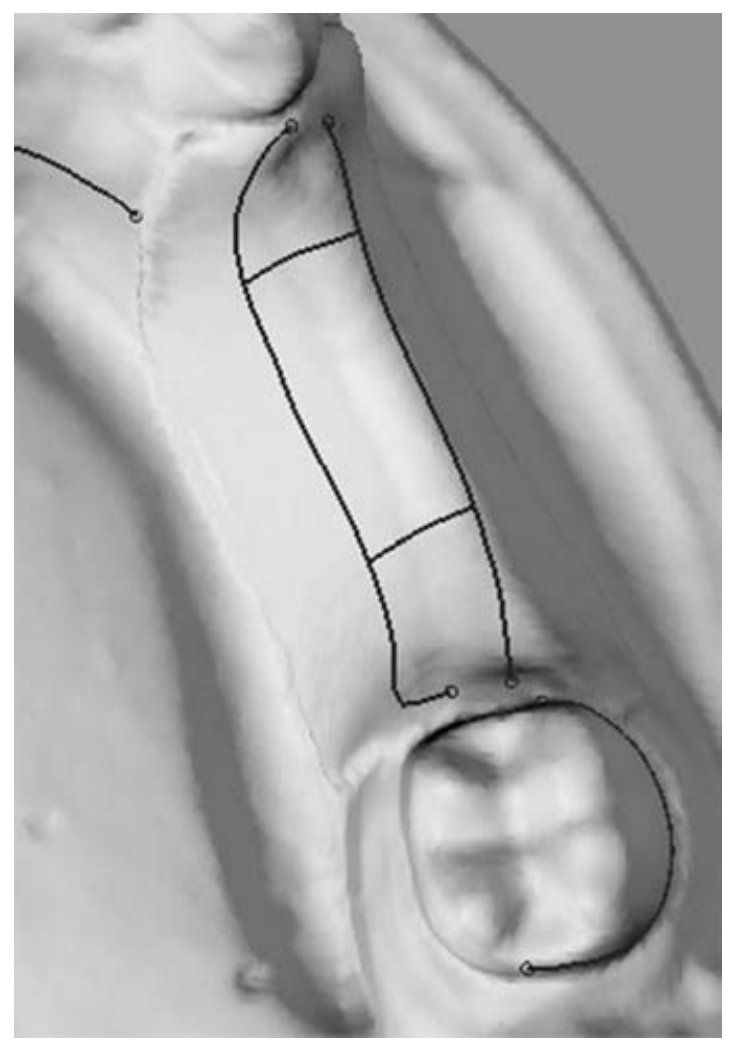

Fig. 6 Construction curves 


\subsubsection{Active clasps}

The clasps were designed in the same manner as the non-flexible parts of the framework, but using the 'buck' cast with undercuts. The construction lines were joined to the termination point previously marked in the undercut measurement stage.

The 'buck' cast was removed leaving the clasps. These were joined to the main framework and blended in. Figure 7 shows the final virtual design. The entire framework was exported as an STL file.

\subsection{Pattern manufacture}

Four RP methods were compared: stereolithography (SL) (3D Systems Inc., Valencia, California, USA), ThermoJet $^{\circledR}$ (3D Systems Inc.), Solidscape ${ }^{\circledR}$ T66 (Solidscape Inc., Merrimack, New Hampshire, USA), and Perfactory ${ }^{\circledR}$ (Envisiontec GmbH, Marl, Germany). Two SL resins were compared: DSM Somos $^{\circledR} 10110$ (Waterclear ${ }^{\mathrm{TM}}$, New Castle, Delaware, USA) and Accura ${ }^{\mathrm{TM}}$ Amethyst $^{\circledR}$ (3D Systems Inc.). Both of the SL patterns were an epoxy-based polymer, the ThermoJet ${ }^{\circledR}$ was TJ88-grade wax polymer, the Solidscape ${ }^{\circledR}$ was a soft thermoplastic, and Perfactory ${ }^{\circledR}$ was an acrylate-based polymer. The Waterclear $^{\mathrm{TM}}$ and ThermoJet ${ }^{\circledR}$ patterns were manufactured at The National Centre for Product Design and Development Research, Cardiff, and the others were prepared and built by external suppliers. The Amethyst, Solidscape ${ }^{\circledR}$, and Perfactory ${ }^{\circledR}$ materials are used by the jewellery industry to produce sacrificial patterns.

\subsubsection{SLA-250 in the Waterclear ${ }^{\mathrm{TM}}$ example}

The STL framework design was prepared using Lightyear $^{\mathrm{TM}}$ (3D Systems Inc.) with a 'fine point' sup-

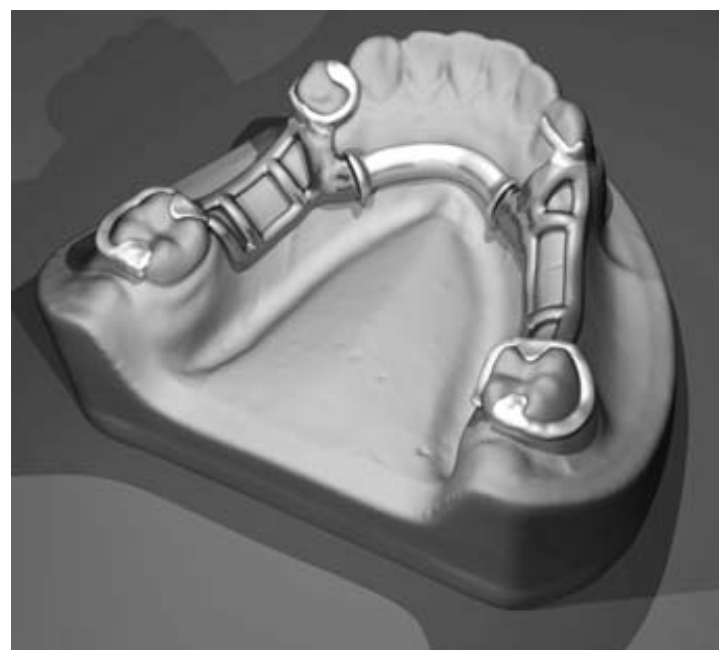

Fig. 7 The complete FreeForm ${ }^{\circledR}$ design port structure (Fig. 8). The framework was oriented with the fitting surfaces facing upwards to avoid the rough finish created by the support structures affecting fit.

Two build styles were compared: standard layers $0.1000 \mathrm{~mm}$ thick and high-resolution layers $0.0625 \mathrm{~mm}$ thick. Once completed, the patterns were carefully removed from the machine platform and cleaned in isopropanol. They were then post cured in ultraviolet light to ensure full polymerization. The other patterns were produced according to the supplier specifications.

\subsection{Pattern comparison}

Of the four RP processes compared in this study, the SL processes provided the most suitable patterns. The SL patterns were accurate and robust and had an acceptable surface finish but did require relatively lengthy cleaning and finishing to remove support structures. The ThermoJet ${ }^{\circledR}$ build preparation was simpler and faster than SL and both the ThermoJet ${ }^{\circledR}$ and the Solidscape ${ }^{\circledR}$ processes produced accurate patterns with a good surface finish that required minimal finishing. These wax patterns were, however, extremely fragile and could not be cast. The Perfactory ${ }^{\circledR}$ produced pattern showed a very smooth surface finish but was also extremely flexible and was easily distorted when handled.

\section{CASTING}

The SL and Perfactory ${ }^{\circledR}$ patterns were cast in chromium-cobalt alloy without using a refractory cast. A slow mould heating cycle was used to avoid cracking. Figure 9 shows the unfinished cast from the SL Amethyst ${ }^{\circledR}$ pattern. This shows that air inclusions from the casting process did not adhere to the pattern surface.

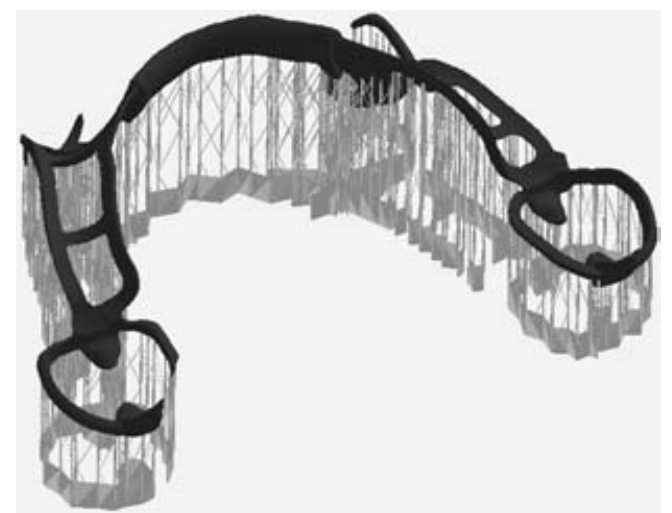

Fig. 8 The support structure in Lightyear ${ }^{\mathrm{TM}}$ 


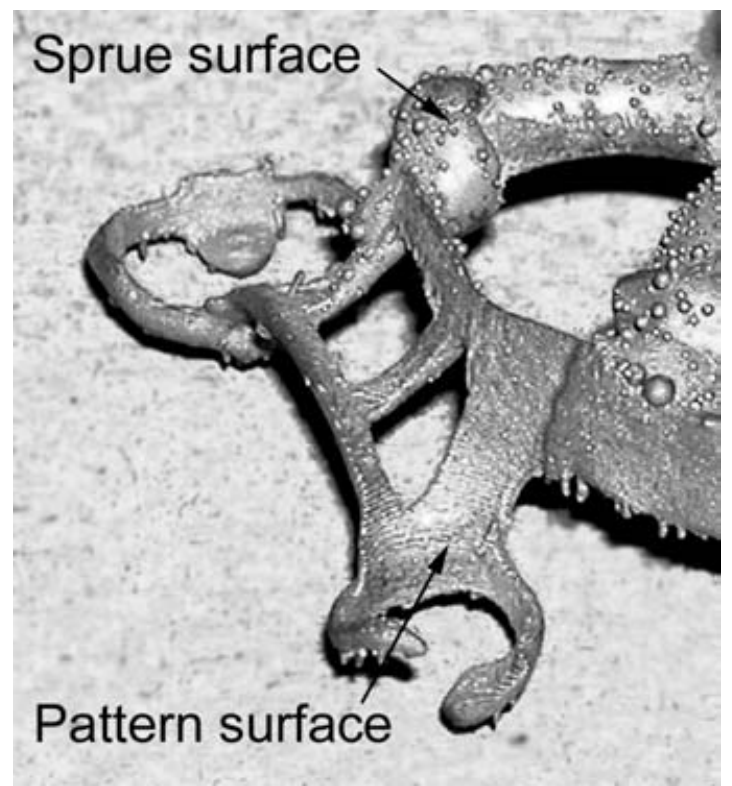

Fig. 9 Surfaces of the unfinished Amethyst ${ }^{\circledR}$ pattern cast

Although casts were obtained from the SL and Perfactory ${ }^{\circledR}$ patterns, it proved difficult to add sprues due to the thin framework sections. In order to improve casting, the design was thickened in FreeForm ${ }^{\circledR}$ and revised SL patterns were produced and cast. This improved the pattern's strength and the casting reliability.

\subsection{Finishing}

The casts produced from the original thin Amethyst ${ }^{\circledR}$ and thicker Waterclear ${ }^{\mathrm{TM}}$ patterns were polished and test fitted to the original physical cast. These were all visually assessed and judged to be satisfactory. Figure 10 shows the finished RPD framework that

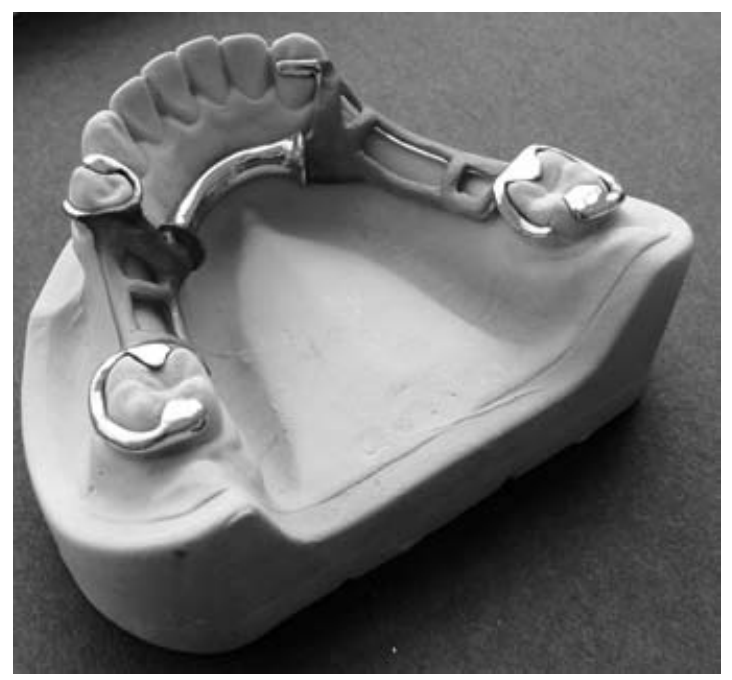

Fig. 10 The definitive framework was cast from the high-resolution Waterclear ${ }^{\mathrm{TM}}$ SLA-250 pattern.

\section{RESULTS}

\subsection{Assessment of the RPD frameworks}

Surprisingly few studies have discussed the accuracy of fit of RPD frameworks [11-13] and even fewer have attempted to quantify it. One study which did attempt to measure gaps between frameworks and tooth surfaces in crucial areas using feeler gauges found that, owing to the three-dimensional nature of the curved surfaces examined, the method may be flawed [13]. Thus researchers routinely rely on the same somewhat subjective assessments which practitioners use on fitting RPD frameworks to patients. For example, occlusal rests are pressurized to detect whether there is movement, the closeness of the adaptation of clasps to teeth is studied, and the alloy surface is checked for visible defects [14].

\subsection{Sources of error}

Error may be produced in all aspects of dental technology if the original impression of the patient's teeth and surrounding tissues is poorly taken or the cast produced from it badly made. Various studies have aimed to assess this error [12-14]. However, in the absence of an appropriate intra-oral scanning technology the application of CAD/CAM in dental technology depends on the dental model. Other than human error in the interpretation of the instructions of the dentist or in the design of the framework, the adoption of CAD/CAM and RP technologies may incur several processes that may contribute to error between the theoretical design and the final manufactured item. For the most part the effect of these processes will be an accumulation of tolerances at each technology stage. However, certain levels of care and skill may still affect the accuracy of these computer-controlled techniques.

The flow chart in Table 1 indicates the steps in the process investigated here and indicates nominal tolerances associated with the various technologies. The accumulation of the tolerances leads to the maximum error that could be expected to result from the technologies alone assuming no human error is encountered. As human skill level and error cannot be assigned a numerical value and may range from zero to complete failure, discussion of this is not included here. However, as this study aims to investigate the implications of adopting CAD/CAM and RP technologies, it is appropriate to attempt to illustrate 
Table 1 Sources of error and tolerances for the steps in the investigated process

\begin{tabular}{lll}
\hline Process step & Source of error & Tolerance \\
\hline Impression taking & Human/skill level & No value \\
Casting study model & Human/skill level & No value \\
Optical scanning of study model & Scanner & $\pm 0.050 \mathrm{~mm}$ \\
Creating polygon computer model from point cloud data & Software & $\pm 0.050 \mathrm{~mm}$ \\
Import into CAD software & Software & $0.000 \mathrm{~mm}$ \\
Design in CAD software & Software & $\pm 0.001 \mathrm{~mm}$ \\
Export of CAD data in STL file format & Software & $\pm 0.010 \mathrm{~mm}$ \\
Physical manufacture using RP process (e.g. SL) & RP machine & $\pm 0.100 \mathrm{~mm}$ \\
Removal of RP pattern from machine, cleaning, and support removal & Human/skill level & No value \\
Preparation for casting, adding sprue, and runner & Human/skill level & No value \\
Casting & Material used & $\pm 0.100 \mathrm{~mm}$ \\
Removing casting sprue and runner & Human/skill level & No value \\
Surface preparation and polishing & Human/skill level & No value \\
Total & & $\pm 0.311 \mathrm{~mm}$ \\
\hline
\end{tabular}

their potential contribution to error in the final RPD. The tolerances used in this table indicate typical or nominal figures, which are quoted by manufacturers or set as parameters in software.

\subsection{Error analysis}

For this application, it is difficult to achieve an investigation that provides detailed quantitative analysis of error. The natures of the devices mean that they are complex in form and do not provide convenient datum or reference surfaces. In addition, the devices are by definition one-off custom-made appliances constructed to fit individual patients. Therefore, it is not practical to perform the type of repeated statistical analysis that would be commonly encountered in series production or mass manufacture. It is normal dental practice to assess the accuracy of an RPD by test fitting the device to the study model and subsequently to the patient. In this study the RPD frameworks created were deemed by a qualified and experienced dental technician to be a satisfactory fit and comparable with those produced by expert technicians.

The parameters set in software are often user selected, are usually set to extremely small fractions of a millimetre, and in all practical terms may be ignored. The significant errors are likely to be encountered at the optical scanning stage and the RP manufacturing stage. Through extensive experience over many years, these figures are frequently encountered in industrial applications of these technologies and as such may be considered typical. The cumulative effect of these tolerances remains submillimetre and as such is likely to be equivalent to or smaller than the typical human error encountered in the traditional dental technology laboratory. The remaining sources of error are encountered in traditional practice and the use of computer-aided technologies will not affect them greatly.

\section{CONCLUSIONS}

The design stages of this technique rely on having an accurate three-dimensional scan of a patient cast and an understanding of both RPD framework design and CAD techniques. This meant that the time taken to produce castable patterns using the technology described is considerable but would be significantly reduced with familiarity and practice.

The most suitable choice of RP process was determined primarily by accuracy and part strength. The ThermoJet $^{\circledR}$ and Solidscape ${ }^{\circledR}$ patterns, although accurate, were too fragile and were therefore not suitable for the tasks associated with spruing and casting. Although the Perfactory ${ }^{\circledR}$ pattern cast well, the accuracy was poor because of distortion inflicted on the flexible pattern during handling. The stiffer patterns produced by SL were easy to handle, were accurate, and produced satisfactory results. The layer effect exhibited by all RP processes was not evident after finishing and the difference between the high-resolution and standard SLA-250 patterns was negligible.

The techniques undertaken and described above outline a stage in the development of machineproduced RPD frameworks and point to many possible advances that can be achieved in the future. The application of CAD would allow access to new RP technologies that build parts directly in metal alloys, including chromium-cobalt and stainless steel. Sacrificial pattern manufacture and casting may be eliminated all together. This will be explored in future studies. 
The introduction of digital design and RP production into current practices would present a significant change in the field of dentistry and is unlikely to happen quickly. Studies so far have shown how CAD and RP may be applied and some principles have been developed and established. Possible future benefits and the potential shortfalls have also been discussed.

\section{ACKNOWLEDGEMENTS}

The authors would like thank Frank Cooper at the Jewellery Industry Innovation Centre in Birmingham, UK, who kindly supplied the Perfactory ${ }^{\circledR}$ and Solidscape ${ }^{\circledR}$ RP patterns and Kevin Liles at 3D Systems Inc. who supplied the Amethyst ${ }^{\mathrm{TM}} \mathrm{RP}$ pattern.

\section{REFERENCES}

1 Hughes, C. W., Page, K., Bibb, R., Taylor, J., and Revington, $\mathbf{P}$. The custom-made titanium orbital floor prosthesis in reconstruction for orbital floor fractures. Br. J. Oral Maxillofacial Surg., 2003, 41, 50-53.

2 Bibb, R. and Brown, R. The application of computer aided product development techniques in medical modeling. Biomed. Sci. Instrum., 2000, 36, 319-324.

3 Mitsuhiro, T., Nobuhiro, N., Koichiro, I., et al. Fabrication of a maxillofacial prosthesis using a computer-aided design and manufacturing system. J. Prosthodontics, 2004, 13(3), 179-183.

4 Willer, J., Rossbach, A., and Weber, H. P. Computerassisted milling of dental restorations using a new CAD/CAM data acquisition system. J. Prosthetic Dentistry, 1998, 80(3), 346-353.
5 Van der Zel, J., Vlaar, S., de Ruiter, W., and Davidson, C. The CICERO system for CAD/CAM fabrication of full ceramic crowns. J. Prosthetic Dentistry, 2001, 85, 261-267.

6 Duret, F., Preston, J., and Duret, B. Performance of CAD/CAM crown restorations. J. Calif. Dent. Assoc., 1996, 9(9), 64-71.

7 Williams, R., Bibb, R., and Rafik, T. A technique for fabricating patterns for removable partial denture frameworks using digitized casts and electronic surveying. J. Prosthetic Dentistry, 2004, 91(1), 85-88.

8 Williams, R., Eggbeer, D., and Bibb, R. CAD/CAM in the fabrication of removable partial denture frameworks: A virtual method of surveying 3-dimensionally scanned dental casts. Quintessence J. Dent. Technol., 2004, 2(3), 268-276.

9 Bibb, R., Freeman, P., Brown, R., Sugar, A., Evans, P., and Bocca, A.. An investigation of threedimensional scanning of human body surfaces and its use in the design and manufacture of prostheses. Proc. Instn Mech. Engrs, Part H: J. Engineering in Medicine, 2000, 214, 589-594.

10 Budtz-Jorgensen, E. and Bocet, G. Alternate framework designs for removable partial dentures. J. Prosthetic Dentistry, 1998, 80, 58-66.

11 Ali, M., Narin, R. I., Sherriff, M., and Waters, N. E. The distortion of cast cobalt-chromium alloy partial denture framework fitted to a working cast. J. Prosthetic Dentistry, 1997, 78(4), 419-424.

12 Stern, M. A., Brudvik, J. S., and Frank, R. P. Clinical evaluation of removable partial denture rest seat adaptation. J. Prosthetic Dentistry, 1985, 53(5), 658662.

13 Murray, M. D. and Dyson, J. E. A study of the clinical fit of cast cobalt-chromium clasps. J. Dentistry, 1988, 16(3), 135-139.

14 Barsby, M. J. and Schwaz, W. D. The qualitative assessment of cobalt-chromium casting for partial dentures. Br. Dent. J., March 1989, 166, 211-216. 